\title{
Rasburicase for Huge Hepatocellular Carcinoma with Tumor Lysis Syndrome: Case Report
}

\author{
Chia-Ter Chao Chih-Kang Chiang \\ Department of Internal Medicine, National Taiwan University Hospital, College of Medicine, \\ National Taiwan University, Taipei, Taiwan
}

\section{Key Words}

Hepatocellular carcinoma - Tumor lysis syndrome •

Rasburicase $\cdot$ Uric acid $\cdot$ Acute kidney injury

\begin{abstract}
Objective: To report a case of huge hepatocellular carcinoma associated with tumor lysis syndrome and its management. Clinical Presentation and Intervention: A 51-yearold hepatitis B carrier visited our clinic with progressive weight loss over recent months. Abdominal ultrasonography and magnetic resonance imaging revealed a huge hepatic mass in a cirrhotic liver, identified as hepatocellular carcinoma. A hepatologist and surgeon recommended transarterial chemoembolization followed by hepatectomy. The patient underwent the procedure, with the complication of tumor lysis syndrome. He was treated with a single high dose $(9 \mathrm{mg})$ of rasburicase and his clinical condition improved dramatically, with acute kidney injury subsiding without emergent hemodialysis. Conclusions: This was a case of hepatocellular carcinoma with tumor lysis syndrome and acute kidney injury, treated successfully with rasburicase.
\end{abstract}

Copyright $\odot 2012$ S. Karger AG, Basel
(C) 2012 S. Karger AG, Basel

1011-7571/12/0215-0498\$38.00/0

Fax +4161306 1234

E-Mail karger@karger.ch

www.karger.com
Accessible online at: www.karger.com/mpp

\section{Introduction}

Tumor lysis syndrome (TLS) occurs in a variety of clinical settings, mostly involving hematologic malignancies such as leukemia, aggressive lymphoma, or rapidly growing solid tumors. It can occur spontaneously following depletion of nutrients within a tumor mass, or be preceded by antitumor therapy [1]. Here, we describe a rare case of huge hepatocellular carcinoma associated with TLS and acute kidney injury.

\section{Case Report}

A 51-year-old Asian man was referred to our clinic for weight loss of $10 \mathrm{~kg}$ in recent months. He had been a hepatitis B carrier since his twenties without regular follow-up, but otherwise denied any medical diseases or surgical history. Upon examination, his vital signs were normal, but abdominal palpation revealed a painless abdominal mass over the right upper quadrant. His $\alpha$-fetoprotein level was normal $(5.25 \mu \mathrm{g} / \mathrm{l}$, ref. $<20 \mu \mathrm{g} / \mathrm{l})$, whereas serum hepatitis B viral load was $5.53 \times 10^{6} \mathrm{IU} / \mathrm{ml}$. Other hepatitis virus serologies were all negative. Abdominal ultrasonography revealed a huge hepatic mass in a cirrhotic liver. Magnetic resonance imaging (MRI) identified a hepatic mass of heterogeneous intensity, $17 \mathrm{~cm}$ in diameter, nearly devouring the right lobe (fig. 1), with hyperintensity upon arterial and venous phase washouts. Hepatocellular carcinoma was diagnosed. A multidisciplinary oncology 
panel suggested transarterial chemoembolization (TACE) for tumor reduction followed by right hepatectomy. He then received TACE over the right hepatic artery (day 1), but spiking fever and severe abdominal pain with oliguria occurred soon after (fig. 2). The blood test results were as follows: hyperphosphatemia: 2.13 $\mathrm{mmol} / \mathrm{l}$; hyperuricemia: $892 \mu \mathrm{mol} / \mathrm{l}$, and acute kidney injury (baseline creatinine 115-362 $\mu \mathrm{mol} / \mathrm{l}$ ). The urinalysis showed signs of mild hematuria (2+) and amorphous urate crystals (3+). The acute kidney injury was unresponsive to allopurinol and aggressive hydration with urine alkalinization. He then became orthopneic. Due to his concern regarding the risks of hemodialysis, we elected to give him single-dose rasburicase $9 \mathrm{mg}(0.15 \mathrm{mg} / \mathrm{kg}$, body weight $60 \mathrm{~kg}$ ). His urine output dramatically increased after day 2 , and orthopnea gradually subsided, with serum creatinine level also decreasing without emergent dialysis. His uric acid level subsequently rose to $6.4 \mathrm{mg} / \mathrm{dl}$ and remained stable 1 week later (fig. 2). He was discharged 2 weeks after this episode and prepared for surgery on his shrinking hepatic tumor.

\section{Discussion}

This was a case of a huge hepatocellular carcinoma with TLS and acute kidney injury managed conservatively with rasburicase without resorting to dialysis. The accompanying hyperuricemia was believed to be due to TLS and acute kidney injury, and the use of rasburicase successfully reversed its course of renal failure.

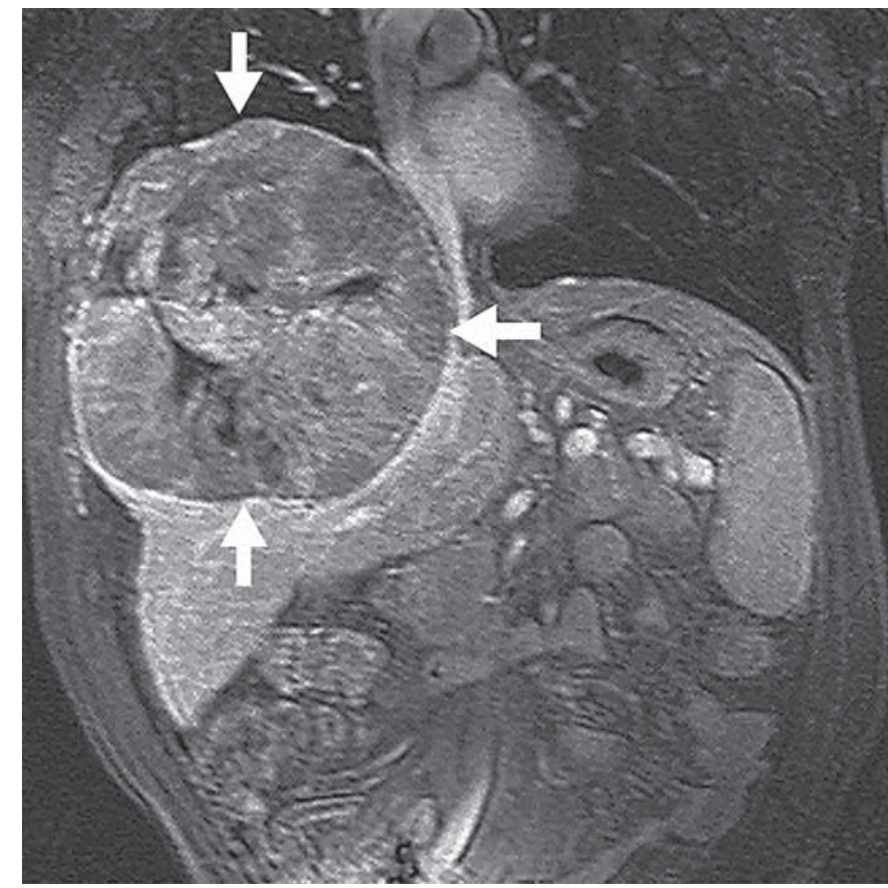

Fig. 1. $T_{1}$-weighted abdominal MRI imaging demonstrates the huge hepatocellular carcinoma (arrows), bulging from the right hepatic lobe and protruding against the right hemi-diaphragm.
Fig. 2. Diagram showing the trends of serum creatinine, uric acid, urine output, and body temperature changes over the treatment course. Rasburicase exerts a rapid effect on acute kidney injury reversal, manifesting itself in an immediate increase in urine output.

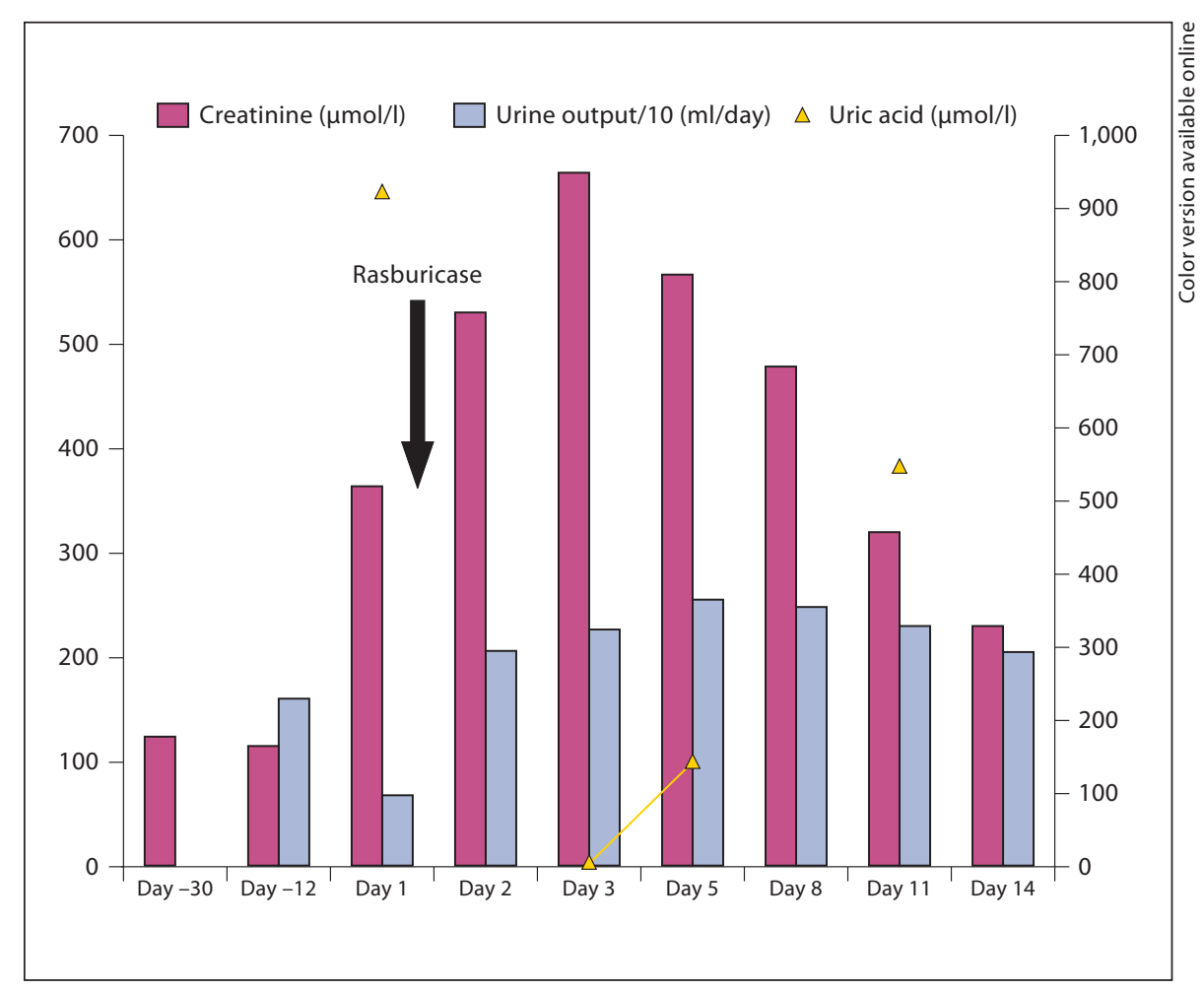


TLS is a catastrophic complication associated with cytotoxic chemotherapy for rapidly growing tumors. The TLS can lead to multiple organ failure, including acute kidney injury, as in this case, seizure, or death from arrhyth mia. Among these, acute kidney injury from hyperuricemia and hyperphosphatemia is pathophysiologically critical. Hyperuricemia can precipitate uric acid crystals within the renal tubules accompanying metabolic acidosis from cell lysis. Hyperphosphatemia also precipitates calcium phosphate crystals, resulting in tubular obstruction and nephrocalcinosis.

TLS can be classified according to the Cairo and Bishop scheme [1], defined as 'laboratory' or 'clinical' TLS, using serum uric acid, phosphate, potassium, calcium, creatinine and clinical parameters. Our patient's presentation matched the 'clinical' TLS category, with oligoanuria and elevation of serum creatinine. Risk factors for developing TLS include tumor types, treatment-related aspects, and patient-level considerations [1]. Tumors of aggressive growth type, a high tumor burden and highly effective therapeutic modality carry the highest risk. Patients with chronic kidney disease, dehydration and hypotension are more susceptible to the development of TLS. Because of our patient's huge hepatocellular carcinoma $(17 \mathrm{~cm})$, the TACE he received, and the contrast medium used during angiography could have potentially led to massive tumor necrosis/parenchymal damage and the nephrotoxic insult.
Solid tumors are rarely associated with TLS and are often linked to a specific treatment modality [1]. The TLS following solid tumor treatment is reportedly distinct from the one following chemotherapy for hematologic malignancy, in both onset and overall prognosis. The former tends to occur days after treatment, rather than hours as in the latter. The prognosis is also poorer in solid tumors, due to delayed presentation. A total of 10 cases of hepatocellular carcinoma presenting with TLS and acute kidney injury has been reported [2-10]. Approximately $60 \%$ are preceded by TACE as in this case.

Rasburicase is approved for the prevention of TLS in patients with high risk features. In our patient, the severe acute kidney injury and the rapid recovery of renal function suggested that rasburicase could be highly effective even in advanced TLS. Considering the potential healthcare saving (dialysis equipment and staff rotation) and reduction of patient anxiety, we suggest that rasburicase can be considered before implementing emergent hemodialysis in carefully selected patients with similar presentations.

\section{Conclusion}

This was a case of hepatocellular carcinoma with TLS and acute kidney injury, treated successfully with rasburicase.

\section{References}

$\checkmark 1$ Howard SC, Jones DP, Pui CH: The tumor lysis syndrome. N Engl J Med 2011;364: 1844-1854.

2 Burney IA: Acute tumor lysis syndrome after transcatheter chemoembolization of hepatocellular carcinoma. South Med J 1998;91: 467-470.

3 Lehner SG, Gould JE, Saad WE, Bronw DB: Tumor lysis syndrome after radiofrequency ablation of hepatocellular carcinoma. AJR Am J Roentgenol 2005; 185:1307-1309.

$\checkmark 4$ Lee CC, Wu YH, Chung SH, Chen WJ: Acute tumor lysis syndrome after thalidomide therapy in advanced hepatocellular carcinoma. Oncologist 2006;11:87-88.
Mukherjee E, Mukherji D, Jayawardene SA, Kon SP: Tumor lysis syndrome and acute renal failure - an increasing spectrum of presentations. Clin Nephrol 2007;68:186-189.

-6 Sakamoto N, Monzawa S, Nagano H, Nishizaki H, Arai Y, Sugimura K: Acute tumor lysis syndrome caused by transcatheter oily chemoembolization in a patient with a large hepatocellular carcinoma. Cardiovasc Intervent Radiol 2007;30:508-511.

7 Shiba H, Ishida Y, Wakiyama S, Sakamoto T, Misawa T, Yanaga K: Acute tumor lysis syndrome after transarterial chemoembolization for hepatocellular carcinoma. Cancer Sci 2008;99:2104-2105. $\checkmark 8$ Huang WS, Yang CH: Sorafenib induced tumor lysis syndrome in an advanced hepatocellular carcinoma patient. World J Gastroenterol 2009;15:4464-4466.

9 Hsieh PM, Hung KC, Chen YS: Tumor lysis syndrome after transarterial chemoembolization of hepatocellular carcinoma: case reports and literature review. World J Gastroenterol 2009; 15:4726-4728.

10 Shiozawa K, Watanabe M, Takenaka H, Nagai H, Ishii K, Sakai K, Sumino Y: Tumor lysis syndrome after sorafenib for hepatocellular carcinoma: a case report. Hepatogastroenterology 2010;57:688-690. 